\title{
Las pobrezas de las juventudes: análisis de las formas elementales de pobreza juvenil en España ${ }^{1}$
}

\author{
FRANCESC VALLS FONAYET ${ }^{2}$ \\ Universitat Rovira i Virgili \\ francesc.valls@urv.cat
}

Recibido: 26.04 .2010

Aceptado: 12.11 .2010

\section{EL ESTUDIO DE LA POBREZA Y LA EXCLUSIÓN SOCIAL JUVENIL EN ESPAÑ}

Distintas voces alertan de la escasez de investigaciones sobre la pobreza y la exclusión social entre los jóvenes en España (París et al. (2006), Comas (2007) y Tully et al. (2008).), aunque, ciertamente, en los últimos diez años ha aumentado el volumen de publicaciones destinadas a este objeto de estudio.

En unas de las obras impulsoras de este campo de estudio, Cantó y Mercader-Prats (1999, 2001a y 2001b) constatan el efecto ayuda que representa la presencia de jóvenes trabajadores en los hogares con riesgo de pobreza, apuntando a una solidaridad bidireccional entre padres y jóvenes. Una estrategia que, como apunta Ayllón (2009), resulta simbiótica: los jóvenes trabajadores encuentran en la familia la protección para hacer frente a las condiciones desfavorables que sufren en el mercado de trabajo, y las familias se benefician de la entrada de una nueva fuente de ingresos. En esta línea, Ayllón et al. (2003) apuntan al elevado riesgo de pobreza que sufren los hogares donde un joven menor de 30 años que vive con sus padres se ha convertido en el sustentador principal -o único- del hogar. Iacovou y Aassve (2007) demuestran que la tasa de pobreza juvenil en España sería superior si la edad de emancipación fuera similar a la de otros países

1 La propuesta metodológica y los resultados publicados en este artículo se inscriben en el marco de la investigación «Nueva pobreza y exclusión social en los jóvenes de España», financiada por el Ministerio de Ciencia e Innovación - Plan Nacional I+D+I (2008-2011) / Proyecto de investigación fundamental no orientada.

Francesc Valls Fonayet. Facultad de Ciencias Económicas y Empresariales. Universitat Rovira i Virgili. Av. Universitat, 1. Reus (43204). Teléfono: 977759896. Correo electrónico: francesc.valls@urv.cat.

EMPIRIA. Revista de Metodología de Ciencias Sociales. N. ${ }^{\circ}$ 21, enero-junio, 2011, pp. 97-120 ISSN: $1139-5737$ 
europeos con otras tipologías de Estado de Bienestar, especialmente en relación a los países nórdicos. En Gómez (2008) se apoya esta hipótesis: los jóvenes españoles que viven en el hogar de origen sufrirían unas elevadas tasas de pobreza si se emanciparan. Albert y Davia (2007), por su parte, indican que el riesgo de pobreza entre los jóvenes se dispara cuando se dan situaciones de precariedad laboral (temporalidad y paro) o de inactividad, entre los jóvenes con bajos niveles de estudios, entre los que viven solos, tienen hijos o que conviven en hogares con tres generaciones.

La profundización del estudio sobre la juventud (así como de otros colectivos vulnerables) es una de las expansiones que ha vivido el ámbito de estudio de la pobreza y la exclusión social en España en los últimos años. Existen otras tres de importante calibre que, a nivel metodológico, debemos citar: a) la armonización respecto a las directrices metodológicas de EUROSTAT $^{3}$; b) la incorporación de investigaciones multidimensionales que superan la idea de pobreza monetaria (Martínez, 2007; Ayala y Navarro, 2007; Maestro y Martínez, 2003; Pérez-Mayo y Fajardo, 2003; Pérez-Mayo, 2003; Martínez y Ruiz-Huerta, 2000; Martín-Guzmán et alt., 2001), y c) el análisis territorial de la pobreza, bien se tenga una visión conjunta del país (Ruíz-Huerta et alt., 1995; EDIS et alt., 1998; Ayala y Martínez, 2005) o bien se centralice en territorios concretos (con una abundantísima producción a nivel local y regional).

Debemos remarcar que, aún con esta progresiva generalización, siguen existiendo limitaciones en el estudio de la pobreza y la exclusión social juvenil, entre las cuales dos se mueven específicamente en un plano conceptual-metodológico:

Por una parte, la homogeneidad en el tratamiento metodológico (dominando lo cuantitativo y lo centrado en las variables monetarias) y disciplinar (economicismo). Cabe señalar que esta limitación se está viendo progresivamente solventada por la incorporación de nuevas aproximaciones. Por ejemplo, por investigaciones cualitativas sobre la vulnerabilidad subjetiva a raíz de la precariedad laboral (Albaigés, 2003), de las dificultades en la inserción a la vida adulta (Cardenal, 2006) o de las desigualdades por razón de género en las conductas de riesgo juveniles (Berga, 2007). Otras investigaciones abordan la pobreza juvenil desde ámbitos no exclusivamente monetarios: Mercader-Prats (2005) apunta al hecho de que cuando el sustentador del hogar es joven (emancipado) la vulnerabilidad no se expresa tanto en términos de pobreza monetaria sino en deficiencias en la capacidad de consumo y en la vivienda, mientras que Ayala (2008) indica que las reducidas tasas de pobreza no esconden una mayor vulnerabilidad subjetiva y un deterioro de las condiciones de vida de los jóvenes.

Por otra parte, la definición del objeto de estudio -los jóvenes- se aborda en la práctica totalidad de las obras citadas a partir de una definición que sitúa la edad biológica, variable soñada, estadísticamente neutra y perfecta (Thévenot,

\footnotetext{
${ }^{3}$ Para un anàlisis de esta transición, ver: Adiego y Moneo, 2004; INE, 2006.
} 
1979), como característica sustancial de un supuesto colectivo social, sin abordar qué factores sociales de disgregación aumentan o disminuyen las posibilidades de sufrir vulnerabilidad entre los distintos grupos sociales de jóvenes. En este punto hay que mencionar la entrada con fuerza del estudio de los itinerarios de transición a la vida adulta (Casal, 1996; Casal et alt., 1988, 2004, 2006; Cardenal, 2006; Miret et alt., 2008), que suponen un paso adelante en lo que refiere a la definición social del objeto de estudio. No obstante, la noción de estructura social sigue siendo una de las principales ausencias en el campo de estudio de la juventud, siendo suplantada aún de forma constante -en su papel de definición de los colectivos sociales- por la variable edad (Martín Criado, 1997, 1998).

\section{PROPUESTA METODOLÓGICA ALTERNATIVA: EL ESTUDIO DE LA VULNERABILIDAD SOCIAL EN LAS JUVENTUDES}

La propuesta metodológica que aquí se presenta pretende impulsar una respuesta desde la disciplina sociológica a estas limitaciones existentes en el campo de estudio de la vulnerabilidad social juvenil. ${ }^{4}$

Para ello se ha objetivado la existencia de distintos grupos sociales, una aproximación estrechamente ligada al análisis sociológico, con el fin de romper

${ }^{4}$ Por lo que refiere a la idea de vulnerabilidad social juvenil, concretamos que se han estudiado los conceptos de pobreza, privación multidimensional y vínculo social. Para el primero se ha utilizado la definición generalizada actualmente por Eurostat: un joven será pobre si vive en un hogar con una renta monetaria equivalente inferior al $60 \%$ de la mediana, utilizando la tabla de equivalencia modificada de la OCDE. Para el estudio de las privaciones se han utilizado dos índices de privación (en el ámbito del consumo y en la vivienda) con valores estandarizados de 0 a 100. Para ello se han contabilizado 11 variables dicotómicas, que tomaban el valor 0 cuando no existía privación y el valor 1 cuando el hogar sí sufría privación. Cada variable se ha ponderado según el grado de generalización de la privación, de forma inversa: aquellas variables con menor generalización de la privación tomaban un mayor peso dentro del modelo analítico. La fórmula matemática para este análisis es la siguiente, donde $I_{i}$ es el indicador dicotómico de presencia o no de privación, y $P_{i}$ es el porcentaje de hogares que no sufren una determinada privación:

$$
P_{d}=\frac{\sum_{\mathrm{i}=1}^{\mathrm{n}} I_{i} P_{i}}{\sum_{\mathrm{i}=1}^{\mathrm{n}} P_{i}} \cdot 100
$$

Por último, el vínculo social se ha analizado mediante estos cuatro indicadores: a) índice de asistencia a actividades culturales (en función de la asistencia al cine, teatro y otras actividades culturales, acontecimientos deportivos, y visitas a lugares de interés cultural, y se ha jerarquizado en cuatro categorías: nula participación a lo largo de un mes, participación en alguna actividad, participación en dos actividades, y participación en más de dos actividades); b) índice de contacto con el entorno (con tres categorías en función del contacto y los encuentros con amigos y familiares que no habitan el hogar: contacto o encuentro diario o semanal con familiares y/o amigos; contacto mensual o, por último, contacto muy esporádico de alguna vez al año o inferior); c) variables dicotómicas de vinculación con alguna organización (política, sindical, religiosa, de ocio, humanitaria, profesional, o de voluntariado), y d) variable dicotómica sobre la posibilidad de pedir ayuda a familiares o vecinos en caso de urgencia. 
la homogeneización artificial, a priori y mediante criterios impuestos por el investigador con la que se construye habitualmente el objeto de estudio. Así, la unidad de análisis no ha sido la juventud sino las juventudes ${ }^{5}$ : agrupaciones de individuos que, además de compartir un mismo período cronológico y afrontar un mismo proceso de inserción laboral y de emancipación familiar, comparten una posición social similar. La fuente de datos utilizada para este ejercicio metodológico ha sido la Encuesta de Condiciones de Vida (ECV) del Instituto Nacional de Estadística (INE) del año $2006^{6}$ con una muestra de 8.831 individuos de 16 a 34 años ${ }^{7}$ como población de referencia.

En España existen varias aportaciones que vislumbran la estructuración social en grupos heterogéneos ${ }^{8}$. En el caso que nos ocupa, la detección de los grupos sociales juveniles se ha establecido a partir de dos técnicas estadísticas, el análisis factorial y el análisis de clasificación, y de los procedimientos metodológicos que se resumen a continuación ${ }^{9}$

${ }^{5}$ El uso del plural, juventudes, lo recogemos de la crítica nominalista de Bourdieu, con el argumento que «hablar de los jóvenes como de una unidad social, de un grupo constituido y con unos intereses comunes, y de referir estos intereses a una edad definida biológicamente ya constituye una manipulación evidente. Por lo menos habría que analizar las diferencias entre las juventudes» (Bourdieu, 2002: 165)

${ }^{6} \mathrm{La}$ ECV posee claras potencialidades, como: a) el tamaño de la muestra, el cual permite hacer clasificaciones desagregadas sin caer en una pérdida de representatividad estadística; b) la existencia de información acerca de distintas dimensiones de carácter social: renta, vivienda, trabajo, hogar, salud, etc.), y c) la posibilidad de comparación entre Comunidades Autónomas. Pero a su vez se ve afectada por otras limitaciones que conviene tener presentes. Entre otras, aprovechamos para resaltar las que influyen directamente al estudio de la pobreza: a) la rápida caducidad de los datos a lo largo del proceso de recolección-publicación-análisis, frente al dinamismo de los cambios del contexto social; b) la universalidad de la encuesta, que limita el análisis de colectivos específicos, como en este caso la juventud, a causa de la no inclusión de variables específicas; c) al ser la unidad de análisis el hogar existen dos condicionantes analíticos, a saber: c1) el primero, la exclusión de la muestra de aquellos colectivos que no viven en un hogar privado (individuos sin hogar, usuarios de viviendas colectivas, etc.), cuantitativamente poco numerosos pero cualitativamente esenciales en el estudio de la pobreza, c2) el segundo, las dificultades para dar cuenta de la heterogeneidad de las posiciones sociales en las que se estructura la familia, y d) la utilización de la renta anual como proxy de los recursos económicos familiares, lo cual no permite conocer las pautas de ahorro/gasto familiar ni el volumen de capital económico acumulado de los hogares.

7 El abanico de edades es expresamente amplio, pues se buscaba restringir al mínimo la influencia del investigador en el concepto juventud y dar así un suficiente margen de libertad al modelo estadístico de agrupación para detectar las realidades sociales inherentes a este amplio conjunto de individuos. Se da prioridad, así, a las agrupaciones establecidas por el modelo de clasificación, en perjuicio de la imposición del investigador.

8 Véanse, por ejemplo: Subirats et al. $(1992,2002)$ o Sánchez y Quintana (2005), cuyas estrategias metodológicas han servido de referencia para la construcción de las agrupaciones sociales juveniles que se presentan.

9 Javier Elzo es el autor que más ha desarrollado estos métodos de análisis en la construcción de tipologías juveniles, aunque centrándose en otros ámbitos de estudio, principalmente los estilos de vida, los valores, la religiosidad y los modelos de convivencia familiar. Ver, por ejemplo: Elzo (2006 y 2009).

EMPIRIA. Revista de Metodología de Ciencias Sociales. N. ${ }^{\circ}$ 21, enero-junio, 2011, pp. 97-120. ISSN: $1139-5737$ 
La primera etapa ha consistido en la selección de las variables integrantes del modelo. Este es un paso clave puesto que de los criterios de selección dependerán tanto el número de grupos construidos como su naturaleza. En este caso se han seleccionado 46 variables explicativas de las condiciones de existencia de los jóvenes, y han sido agrupadas previamente en seis dimensiones temáticas: condiciones de vida; vínculo social; territorio y contexto urbano; perfil social y demográfico; familia y composición del hogar y, por último, nivel formativo y posición en el mercado laboral. Todas las variables han sido medidas a nivel nominal u ordinal.

En la segunda etapa se ha realizado, dentro de cada una de estas dimensiones, y de forma independiente, un análisis de componentes principales categóricos con el que se ha conseguido, entre otros requisitos esenciales como la reducción de información, situar diferenciadamente a los individuos respecto a unas nuevas variables latentes, más significativas y que más discriminan a los individuos entre ellos, y que estas nuevas variables fueran linealmente independientes con el fin de no sesgar el posterior análisis de clasificación (la selección del número de variables latentes se basó en el análisis de la varianza total explicada -alrededor del $70 \%$ en todos los casos-, en el número de ejes con autovalores superiores a 1, y en la interpretación sociológica del peso de las variables originales sobre cada variable latente). En la tercera etapa se ha realizado un análisis de conglomerados en dos fases que posibilitó la agrupación de individuos con características similares dentro de cada una de estas seis dimensiones sociales, con lo que se obtuvieron seis nuevas variables de tipo nominal (una por cada dimensión social) con distintas categorías (una por cada grupo social juvenil construido en su interior). Este método de clasificación resulta idóneo cuando se trabaja con una muestra voluminosa y permite una determinación automática del número final de agrupaciones obtenidas (un factor especialmente indicado para análisis descriptivos). En la cuarta etapa se ha llevado a cabo un análisis de correspondencias múltiples a partir de las seis variables nominales obtenidas en el paso anterior (siguiendo los mismos criterios de selección de nuevas variables latentes) para, posteriormente, realizar un último análisis de conglomerados que ha agregado los 8.831 individuos de 16 a 34 años de la muestra en 7 grupos sociales juveniles, que han representado nuestras unidades de análisis. ${ }^{10}$

En la sexta etapa se han validado los resultados obtenidos, mediante tres estrategias: a) el uso del método de replicación, esto es, la repetición del proceso en submuestras con el fin de contrastar la estabilidad de las particiones generadas; b) a través de un análisis de varianza para corroborar estadísticamente las di-

${ }^{10}$ La etapa 4 fue controlada, posteriormente, mediante un nuevo análisis que otorgaba a las dimensiones «perfil sociodemográfico» y «nivel formativo y posición en el mercado laboral» la condición de variables explicativas. El resultado final de esta prueba ha sido la obtención de 4 grupos sociales juveniles, con una identidad similar a la de los grupos obtenidos en el modelo original, pero con una menor nitidez. Por este motivo se explican aquí las particiones del modelo original.

EMPIRIA. Revista de Metodología de Ciencias Sociales. N. ${ }^{\circ}$ 21, enero-junio, 2011, pp. 97-120 ISSN: $1139-5737$ 
ferencias entre los siete grupos sociales juveniles ${ }^{11}$, y c) con la constatación de la relevancia y la coherencia teórica de estos grupos sociales, que han dado luz verde, así, al séptimo y último paso, la definición e interpretación de los grupos sociales juveniles resultantes y el análisis de las formas de vulnerabilidad social asociadas a cada uno de ellos, y que se detallan en el siguiente apartado.

Las tablas 1 y 2 dan cuenta del proceso metodológico detallando los resultados obtenidos en cada etapa.

Tabla 1. Resumen del modelo metodológico (etapas 1 a 3)

\begin{tabular}{|l|c|c|c|c|}
\hline & \multicolumn{1}{|c|}{ Etapa 1 } & \multicolumn{2}{|c|}{ Etapa 2 } & Etapa 3 \\
\hline Dimensión & $\begin{array}{c}\text { Variables } \\
\text { iniciales }\end{array}$ & $\begin{array}{c}\text { Factores } \\
\text { extraídos }\end{array}$ & $\begin{array}{c}\text { \% Varianza } \\
\text { total } \\
\text { explicada }\end{array}$ & $\begin{array}{c}\text { Clústeres } \\
\text { temáticos } \\
\text { obtenidos }\end{array}$ \\
\hline A. Condiciones de vida & 6 & 3 & 68,3 & 2 \\
\hline B. Vínculo social & 9 & 6 & 74,6 & 4 \\
\hline C. Territorio y contexto urbano & 2 & 1 & 68,5 & 3 \\
\hline D. Perfil social y demográfico & 5 & 3 & 84,1 & 2 \\
\hline $\begin{array}{l}\text { E. Familia y composición } \\
\text { del hogar }\end{array}$ & 5 & 3 & 83,4 & 5 \\
\hline $\begin{array}{l}\text { F. Nivel formativo y posición } \\
\text { en el mercado laboral }\end{array}$ & 4 & 2 & 88,3 & 3 \\
\hline
\end{tabular}

Fuente: Elaboración propia a partir de la Encuesta de Condiciones de Vida 2006

Tabla 2. Resumen del modelo metodológico (etapas 4 y 5)

\begin{tabular}{|c|c|c|c|c|}
\hline & $\begin{array}{c}\text { A partir de } \\
\text { la etapa 3 }\end{array}$ & \multicolumn{2}{|c|}{ Etapa 4 } & Etapa 5 \\
\hline Dimensión & $\begin{array}{c}\text { Variables } \\
\text { iniciales }\end{array}$ & $\begin{array}{c}\text { Factores } \\
\text { extraídos }\end{array}$ & $\begin{array}{c}\text { \% Varianza } \\
\text { total } \\
\text { explicada }\end{array}$ & $\begin{array}{c}\text { Clústeres } \\
\text { temáticos } \\
\text { obtenidos }\end{array}$ \\
\hline $\begin{array}{c}\text { Única (a partir de las 6 variables } \\
\text { obtenidas en la etapa 3) }\end{array}$ & 6 & 3 & 73 & 7 \\
\hline
\end{tabular}

Fuente: Elaboración propia a partir de la Encuesta de Condiciones de Vida 2006

${ }^{11}$ Este contraste se realizó mediante nueve variables que daban cuenta de distintas dimensiones de la realidad social juvenil analizada: edad, edad en la que se consigue el máximo nivel formativo, edad de entrada al mercado laboral, renta anual por unidad de consumo, índice de privación en la vivienda, índice de privación en el consumo, volumen de asistencia a actividades culturales en un año, índice de contacto con el entorno, e índice de participación en organizaciones. El análisis post-hoc por pares de categorías confirma que las diferencias entre los grupos son generalizadas. Solamente los grupos 2 (adolescencia rural) y 4 (adolescencia urbana acomodada) en la variable edad, y los grupos 1 (juventud adulta acomodada) y 2 (adolescencia rural), en el índice de privación en la vivienda, presentan unas medias no diferentes con un nivel de significación de 0,01 .

EMPIRIA. Revista de Metodología de Ciencias Sociales. N. ${ }^{\circ}$ 21, enero-junio, 2011, pp. 97-120. ISSN: 1139-5737 
Por último, señalamos que este ejercicio metodológico cuantitativo ha servido de guía para la elaboración de la muestra estructural del análisis cualitativo, con el objetivo de conocer qué discursos entorno a la experiencia vivida de la vulnerabilidad social se generan dentro de cada una de estas agrupaciones sociales de jóvenes. Centrada en los tres grupos sociales claramente vulnerables, la planificación ha sido la siguiente:

Tabla 3. Articulación metodológica. Planificación de la fase cualitativa (entrevistas biográficas), a partir de los resultados obtenidos en la fase cuantitativa de la investigación

\begin{tabular}{|c|c|c|l|}
\hline $\begin{array}{c}\text { Grupo social } \\
\text { juvenil de } \\
\text { referencia }\end{array}$ & $\begin{array}{c}\text { Porcentaje } \\
\text { sobre el } \\
\text { total de los } \\
\text { 3 grupos }\end{array}$ & $\begin{array}{c}\text { Número } \\
\text { de } \\
\text { entrevistas }\end{array}$ & \multicolumn{1}{c|}{ Criterios de selección } \\
\hline $\begin{array}{c}\text { Adolescencia } \\
\text { desfavorecida }\end{array}$ & 42,5 & 13 & $\begin{array}{l}\text { Corta edad (16 a 20 años). Equilibrio de géneros. } \\
\text { Entornos urbanos. Hogar de origen. Rentas eco- } \\
\text { nómicas bajas. Nivel formativo básico. Estudiantes } \\
\text { y parados (u ocupados en categorías poco cualifi- } \\
\text { cadas). Problemas en la vivienda o dificultades en } \\
\text { el consumo. Sin rotura del vínculo social (amigos, } \\
\text { vida social) }\end{array}$ \\
\hline $\begin{array}{c}\text { Juventud } \\
\text { descalificada }\end{array}$ & 31,4 & 9 & $\begin{array}{l}\text { De 20 a 29 años. Equilibrio de géneros. Entornos ur- } \\
\text { banos. Emancipados (o en previsión). Rentas eco- } \\
\text { nómicas bajas. Nivel formativo básico. Ocupados } \\
\text { en el segmento inferior, parados o inactivos (invali- } \\
\text { dez, enfermedades). Problemas de salud. Problemas } \\
\text { en la vivienda o dificultades en el consumo. Aisla- } \\
\text { miento social. Presencia de extranjeros. }\end{array}$ \\
\hline $\begin{array}{c}\text { Juventud } \\
\text { adulta } \\
\text { precarizada }\end{array}$ & 26,1 & 8 & $\begin{array}{l}\text { Adultos (a partir de 26 años). Ligera sobrerrepre- } \\
\text { sentación femenina. Entornos urbanos. Emancipa- } \\
\text { dos. Rentas económicas bajas. Nivel formativo bá- } \\
\text { sico. Ocupados (baja cualificación) y parados. } \\
\text { Presencia de extranjeros. Problemas en la vivienda o } \\
\text { dificultades en el consumo. Baja vinculación social. }\end{array}$ \\
\hline
\end{tabular}

\section{LA REALIDAD SOCIOLÓGICA: LAS SIETE AGRUPACIONES SOCIALES JUVENILES Y LAS FORMAS ELEMENTALES DE LA POBREZA EN ESPAÑA}

\section{Grupo I: La juventud adulta próspera}

La agrupación social más poblada surgida del modelo de clasificación puede, paradójicamente, situarse fuera de lo juvenil. A este grupo social pertenece una de las últimas oleadas de nacidos en la explosión demográfica de los años 70. Se convierte de esta forma en el grupo juvenil más poblado, con alrededor de tres millones de individuos de 16 a 34 años, un $24,2 \%$ del total, y con una media 
de edad entorno a los 30 años. Es, además, el grupo social más feminizado: el $55,2 \%$ de sus miembros son mujeres. Todos estos jóvenes se han emancipado (casi ocho de cada diez son propietarios de su nueva vivienda), y un 63,6\% ya están casados. Su localización territorial y urbanística no difiere de la proporcionalmente esperada, con un dominio de las Comunidades Autónomas (CCAA) de mayor peso demográfico (Cataluña, Andalucía, Comunidad Valenciana y Madrid, por este orden, acumulan el $61 \%$ de estos jóvenes) y de los núcleos altamente urbanizados (51,5\% de los individuos). Existe, además, un contingente de población de nacionalidad extranjera (7,2\% del total): se trata de aquellos jóvenes inmigrantes que han conseguido alcanzar un nivel de vida relativamente acomodado, pudiendo huir así de la precariedad social en la que circulan el resto de los jóvenes de nacionalidad extranjera analizados.

Estos jóvenes fueron los protagonistas, en los noventa, del contingente de población que se tituló en masa en las carreras universitarias. La crisis económica de los primeros años de la década supuso para ellos un estímulo para formarse, estrategia que les llevó a ser, pocos años después, la generación más formada de la historia de España. El 45\% de estos jóvenes ha completado estudios universitarios (casi cuatro de cada diez jóvenes de 16 a 34 años con estudios universitarios pertenecen a este grupo social), y tres de cada cuatro ha superado algún nivel de estudios post-obligatorios. El alargamiento de la carrera formativa es una de las claves para entender su posición acomodada en el mercado laboral: ocho de cada diez jóvenes estaban ocupados, prácticamente todos a tiempo completo y con una generalización de la contratación indefinida. Existe, cabe señalar, una ligera desigualdad por razón de género: entre las mujeres, aunque su posición en el mercado laboral está consolidada (comparándola, sobretodo, con las mujeres de los otros grupos sociales juveniles), ésta sigue siendo, a igual nivel formativo, inferior a la de los hombres, especialmente a partir del momento de la reproducción familiar.

Este grupo presenta la tasa de pobreza más reducida de los siete grupos sociales juveniles: solo un 7,2\% de los jóvenes vivía en un hogar con una renta equivalente inferior al umbral de pobreza. Esto se debe, además de a su elevada formación y su posición acomodada en el mercado de trabajo, a la tipología de hogares en los que viven la mayoría de estos jóvenes: emancipados y en pareja, ya sea con hijos (37\%) o sin ellos (43\%). Especialmente en el segundo caso, se trata de hogares con elevada protección, y más siendo habitual que los dos miembros de la pareja trabajen (como aquí ocurre).

Además de la seguridad ante la pobreza económica, también es el grupo con menor afectación de la privación multidimensional: más del $80 \%$ de los jóvenes de este grupo no se ve afectado por privaciones de carácter grave ${ }^{12}$ y tan sólo un $1,4 \%$ sufre, a la vez, privaciones y pobreza económica.

${ }_{12}$ Para determinar si un hogar sufre un nivel grave de privación multidimensional se han comparado los valores de los índices de privación (en la vivienda y en la capacidad de consumo) de cada hogar con jóvenes con los valores medios. Los hogares con privación grave son aquellos con unos índices de privación en la vivienda y en el consumo superiores, a la vez, al doble de la media.

EMPIRIA. Revista de Metodología de Ciencias Sociales. N. ${ }^{\circ}$ 21, enero-junio, 2011, pp. 97-120. ISSN: 1139-5737 
El vínculo social es resultado de dos factores con fuerzas opuestas: su nivel de vida acomodado facilita el establecimiento de relaciones con el entorno social mientras que su edad avanzada y, especialmente, las responsabilidades asumidas a raíz del establecimiento de una familia de procreación, limitan la disponibilidad de tiempo libre. La consecuencia es la existencia de un vínculo social fuerte en lo que se refiere a las relaciones con familiares y amigos (el contacto es cuotidiano), así como a nivel de participación formal en organizaciones (por encima de la media en todos los tipos de organizaciones, excepto en las orientadas al ocio, típicas de jóvenes de menor edad), mientras que la asistencia a actividades culturales y deportivas sí se ve resentida por la menor disponibilidad de tiempo libre.

\section{Grupo II: La adolescencia rural y la pobreza integrada}

Al segundo grupo con mayor peso demográfico pertenecen jóvenes adolescentes que habitan sobretodo en entornos rurales. A él pertenecen prácticamente 2 millones de españoles de 16 a 34 años (15,5\% del total), con una considerable concentración masculina $(56,1 \%)$. Está formado por jóvenes y adolescentes (siete de cada diez jóvenes tienen menos de 25 años) que viven en el hogar de origen. La característica que distingue en mayor medida a estos jóvenes se refiere al entorno geográfico. El 51,1\% vive en entornos poco urbanizados (el doble de la media juvenil) y sólo un $11,9 \%$ en zonas densamente pobladas (lejos del 52,6\% de la media). Además, más de la mitad vive en viviendas unifamiliares mientras que solamente uno de cada cuatro lo hace en bloques de más de diez viviendas. Este componente rural se explicita también en la localización geográfica: la mayoría de estos jóvenes habitan las CCAA con una menor renta per cápita, especialmente del sur de España (uno de cada tres jóvenes de este grupo vive en Andalucía, la más poblada, seguida de Castilla la Mancha).

Prácticamente la mitad de estos jóvenes son estudiantes. La dimensión formativa no parece representar un obstáculo sino más bien lo contrario: formarse es para estos jóvenes una estrategia defensiva ante el menor abanico de ofertas laborales que les depara su entorno social. Esto es especialmente relevante en el caso de las mujeres, siendo éste el grupo con mayor desigualdad de género en lo que refiere al nivel formativo, en favor de ellas: aún teniendo una menor edad media, el 16\% de las chicas de este grupo ya han completado estudios universitarios (un 44,5\% la secundaria post-obligatoria), frente al 7,1\% de los hombres (45\% la secundaria post-obligatoria). La vinculación laboral aún es lejana y solamente cuatro de cada diez la establecen como su principal ocupación. Además, el período de inserción laboral plena se alarga hasta casi un año y medio (entre la obtención del máximo nivel formativo y la entrada regular al mercado laboral), el período más largo de todos los grupos sociales juveniles, cosa que evidencia las dificultades existentes para la inserción laboral de estos jóvenes. Además, su baja edad se traduce en un elevado impacto de la desocupación y del trabajo temporal.

EMPIRIA. Revista de Metodología de Ciencias Sociales. N. ${ }^{\circ}$ 21, enero-junio, 2011, pp. 97-120 ISSN: $1139-5737$ 
Pero a nivel laboral la principal característica es la desigualdad de género, en este caso en perjuicio de ellas. La tasa de ocupación masculina prácticamente dobla la femenina $(51,2 \%$ y $27,4 \%$, respectivamente), la tasa de paro femenina es la más elevada de todos los grupos sociales (una de cada tres mujeres activas estaba desocupada, más del doble que en los hombres) y, entre las ocupadas, también se reproduce la vulnerabilidad: existe una clara concentración femenina en las ocupaciones a tiempo parcial y en la contratación temporal.

El 17,3\% de los jóvenes de esta clase están en situación de pobreza, una tasa ligeramente superior a la media, aunque esto se debe en mayor medida al hecho de vivir en un entorno de rentas bajas generalizadas que a la acumulación de factores individuales generadores de procesos de vulnerabilidad, como sucede en otras clases sociales juveniles. En este sentido, la pobreza económica no se traduce en una acumulación de dificultades en otros ámbitos sociales: este es el grupo menos afectado por la privación multidimensional y uno de los que presenta un menor riesgo de ruptura de la cohesión social. De esta forma, se puede definir esta vulnerabilidad como una pobreza de tipo integrado ${ }^{13}$ que presenta en estos jóvenes las siguientes características:

Afecta a un volumen considerable de jóvenes con perfiles muy distintos. Por ejemplo, entre los jóvenes con el máximo y el mínimo nivel formativo la diferencia en la tasa de pobreza es de sólo 5 puntos. Por otra parte, estar ocupado, parado, ser inactivo o trabajar en tareas del hogar implica la menor desigualdad en el riesgo ante la pobreza con respecto a los jóvenes ocupados.

No aparece un riesgo de estigmatización social, a causa de la generalización de la pobreza en perfiles a priori distintos: este es el grupo social donde menos familias pobres indican que llegan a final de mes con dificultades (menos de una de cada cuatro), con unos resultados similares a los que muestran las familias no pobres (una de cada cinco).

Tampoco hemos detectado que la pobreza se traduzca en un deterioro de las relaciones sociales. Los vínculos familiares y de amistad son fuertes y frecuentes, y la participación en actividades públicas no se ve resentida por las limitaciones económicas de las familias. En el ámbito laboral existe un importante peso de la economía familiar: uno de cada diez jóvenes ocupados y en situación de pobreza trabajaba ayudando a su familia (trabajo no remunerado explícitamente), lo cual resalta la fuerza de la solidaridad familiar para afrontar situaciones de vulnerabilidad (ningún otro grupo juvenil tiene unos porcentajes rele-

${ }^{13}$ Paugam (2007) distingue tres formas elementales de pobreza: a) la pobreza integrada es característica de sociedades con menor desarrollo económico, con unos entornos de rentas bajas generalizadas y donde el hecho de ser pobre no comporta un riesgo de estigmatización ni de ruptura social, sobre todo gracias a la solidaridad familiar; b) la pobreza marginal es inherente a sociedades industrialmente desarrolladas, y la sufren pequeños grupos de población que no han seguido las normas impuestas por este tipo de organización social, caracterizándose por un deterioro de su imagen social, y c) la pobreza descalificadora es la forma elemental de pobreza característica de las sociedades postindustriales, donde el modelo social y económico pone a amplias capas de población en riesgo de caída hacia procesos de ruptura social. 
vantes en esta variable). Esta protección familiar viene a cubrir las limitaciones de la débil cobertura social: es el grupo de jóvenes no emancipados con menor presencia de protección social pública y con un menor efecto de esta protección ante la pobreza.

\section{Grupo III: La adolescencia desfavorecida como prolongación de la pobreza infantil}

Este grupo social juvenil es el tercero en peso demográfico: prácticamente dos millones de jóvenes españoles pertenecen a él, un 15,3\% del total. Es la más poblada de las clases sociales juveniles que presentan limitaciones en el desarrollo de sus condiciones de vida. Por esto, se erige como un foco de atención principal en la lucha contra la pobreza juvenil: prácticamente uno de cada tres jóvenes de 16 a 34 años en situación de pobreza $(31,8 \%)$ posee estas características. En este caso, se trata de una vulnerabilidad heredada de la familia de origen y que da relevo a las situaciones de pobreza infantil que ya han sufrido anteriormente estos jóvenes.

Es el grupo social con mayor concentración masculina (el 56,4\% de sus miembros son hombres), con una baja edad media (23 años), y con la totalidad de individuos viviendo en el hogar de origen. Uno de cada tres jóvenes habita en CCAA con una renta per cápita inferior al $80 \%$ nacional, siendo Andalucía (con un 26,1\% del total de jóvenes) la más representada, seguida de Cataluña, Comunidad Valenciana y Madrid. A partir de aquí, la proporcionalidad demográfica se rompe y aparecen las CCAA de Canarias y Galicia. Cabe destacar que la totalidad de sus miembros tienen nacionalidad española.

A nivel formativo, una parte importante de estos jóvenes desfavorecidos abandonaron las aulas escolares durante la primera mitad de la década del 2000 para ponerse a trabajar, siendo todavía adolescentes. Aunque la salida del sistema formativo ha sido prematura (se da, de media, a los 17,7 años, edad a la que la mitad de estos jóvenes tienen como máximo la educación secundaria obligatoria), es posible que algunos de ellos, viendo que su posición en el mercado de trabajo difícilmente mejore con las capacitaciones de que disponen, puedan volver al sistema formativo, debido a su aún corta edad. A nivel laboral, los datos confirman que estos jóvenes están conociendo la dureza del segmento inferior del mercado de trabajo: el decalaje entre formación y trabajo es importante (prácticamente de un año y medio), la tasa de paro aumenta hasta el 16,1\% (la más alta de los grupos sociales juveniles), alrededor de dos de cada tres jóvenes ocupados tienen un contrato temporal, y las categorías profesionales mayoritarias son las relacionadas con tareas de ejecución que requieren poca cualificación (restauración, comercio, industrias manufactureras, construcción...) y la mano de obra no cualificada.

La situación de vulnerabilidad se explicita al detectar que prácticamente uno de cada cuatro jóvenes de este grupo $(24,9 \%)$ está en situación de pobreza,

EMPIRIA. Revista de Metodología de Ciencias Sociales. N. ${ }^{\circ}$ 21, enero-junio, 2011, pp. 97-120 ISSN: 1139-5737 
casi 10 puntos por encima de la media juvenil $(15,3 \%)$. Además, si no se contabilizaran las prestaciones sociales de carácter monetario, el grupo de jóvenes desfavorecidos sería el más afectado por la pobreza (con una tasa del 44,6\%). En este sentido, el carácter familiarista de la protección pública en España y el hecho que estos jóvenes todavía habiten en el hogar de origen ayudan a contener el riesgo de pobreza.

La pobreza se agravia en los casos donde los jóvenes no están vinculados al mercado laboral (de aquí la elevada tasa de pobreza femenina, dada su tendencia a prolongar la etapa formativa): el 35,9\% de los jóvenes estudiantes y el 39,7\% de los parados de este grupo padecían una situación de pobreza. Además, estos jóvenes también son los que sufren en mayor medida (con diferencia) privaciones graves de carácter multidimensional (en el $89,4 \%$ de los casos) y es el grupo con una mayor tasa de pobreza femenina (un 28,2\% de las mujeres son pobres).

Aún así, la vulnerabilidad económica (pobreza) y material (privación) no tienen un efecto notable sobre el vínculo social. Los distintos indicadores utilizados para aproximarnos a esta dimensión no muestran que los adolescentes desfavorecidos tengan un comportamiento substancialmente distinto del de los jóvenes con un mayor nivel de vida. En este ámbito se yuxtaponen dos fuerzas que actúan en sentido opuesto. Por un lado, la baja edad de estos adolescentes facilita el contacto con el entorno social: tienen un contacto cuotidiano con amigos (cabe señalar que muchos de estos jóvenes asisten a centros formativos) y familiares. Además, la permanencia en el hogar de origen les libera de las responsabilidades derivadas de la procreación. Por otro lado, la vulnerabilidad social generalizada que sufren (que se expresa en unos elevados índices de pobreza y de privación, pero también en un considerable fracaso escolar y en precariedad laboral) limita sus posibilidades relacionales en aquellas actividades con un coste económico asociado. Cabe señalar, por último, el alejamiento de estos adolescentes desfavorecidos respecto a actividades de un elevado calaje cultural (teatro, conciertos, ópera, visitas a lugares de interés cultural), así como una baja implicación participativa en organizaciones de cualquier tipo, a excepción de aquellas orientadas a actividades de tipo ocioso. La consecuencia de esta relación de fuerzas opuestas es que estos adolescentes desfavorecidos presentan un vínculo social y relacional menos consolidado que el del resto de jóvenes de corta edad (factor vulnerabilidad) pero más dinámico que el de los jóvenes de edad avanzada (factor edad).

\section{Grupo IV: La adolescencia urbana acomodada}

El grupo de adolescentes urbanos acomodados es la cuarta clase social juvenil más poblada, con más de un millón y medio de individuos de 16 a 34 años (13,7\% del total). A ella pertenecen adolescentes que viven en entornos altamente urbanizados (100\% de los jóvenes) de las CCAA con mayor PIB per cápita: Madrid y Cataluña agrupan a más de la mitad de ellos, y junto con la Co- 
munidad Valenciana y el País Vasco engloban a 7 de cada 10 jóvenes de este grupo. En cambio, no forma parte de este grupo ningún joven de las tres CCAA con menor PIB per cápita: Andalucía, Extremadura y Castilla la Mancha, una situación exclusiva.

Estos adolescentes tienen la edad media más baja (22,1 años), con lo cual comparten las características de los otros grupos de adolescentes: permanencia en el hogar de origen, soltería y concentración masculina. Mayoritariamente son estudiantes que han empezado a cursar una carrera universitaria o están a punto de terminar estudios secundarios post-obligatorios. Así, se trata de un perfil de jóvenes con un nivel formativo presente medio y con unas elevadas expectativas de alcanzar niveles formativos superiores en el futuro: con la edad media más baja, estos adolescentes urbanos ya son el tercer grupo social juvenil con un mayor índice de superación de los estudios secundarios post-obligatorios $(64,4 \%$ del total). Esta vinculación formativa ( 9 de cada 10 jóvenes de 16 a 19 años y 2 de cada 3 de 20 a 24 años estaba estudiando) implica una vinculación laboral muy menor (sólo uno de cada tres jóvenes estaba ocupado). Ésta, cuando se da, lleva a los jóvenes a pagar el peaje de la precariedad en los primeros años de trabajo: a) uno de cada cinco jóvenes ocupados lo hacía a tiempo parcial $(20,8 \%$, ocho puntos por encima de la media); b) entre la población activa, uno de cada cinco jóvenes estaba desocupado (tan sólo por detrás de los adolescentes desfavorecidos y de los jóvenes descalificados), mientras que en las mujeres el porcentaje ascendía al 30,1\%, más del doble que en los hombres, y c) 6 de cada 10 jóvenes tenía un contrato temporal, sólo por detrás de los adolescentes rurales y los jóvenes desfavorecidos.

Aún con esta precariedad laboral provisional, los urbanos son el grupo de adolescentes más acomodado, pudiendo disfrutar de un considerable bienestar. Tan solo un 9,1\% de ellos sufría situaciones de pobreza, y se encuentran las tasas de pobreza más bajas en algunos perfiles juveniles: en los jóvenes de hasta 24 años, en los que de momento sólo han superado los niveles más básicos de formación, así como en los ocupados, parados y estudiantes. De hecho, si no es el grupo con menor riesgo de pobreza es sólo debido a su estructura demográfica: la mayoría de individuos se concentra en los perfiles, a priori, más vulnerables (especialmente aquellos alejados del mercado laboral). Cabe añadir que, aún siendo su posición económica ya de principio acomodada, disfrutan del segundo mayor efecto de la protección pública: el riesgo de pobreza una vez contabilizadas las prestaciones monetarias se reduce en un $60,1 \%$.

Estos adolescentes urbanos también tienen una escasa afectación de privación en la mayoría de indicadores. El carácter plenamente urbano de sus viviendas (mayoritariamente en grandes bloques de pisos) garantiza unas condiciones materiales aceptables (aunque, como contrapartida, otros aspectos como el ruido proveniente del exterior, la contaminación o la presencia de delincuencia en el entorno son más habituales en los hogares de estos jóvenes que en la media), mientras que la capacidad económica de sus familias les otorga una evidente acomodación en el terreno del consumo.

EMPIRIA. Revista de Metodología de Ciencias Sociales. N. ${ }^{\circ}$ 21, enero-junio, 2011, pp. 97-120 ISSN: $1139-5737$ 
Esta seguridad en términos económicos y materiales se refleja en la existencia de un vínculo social y relacional consolidado. Asisten con cierta periodicidad a actividades de tipo cultural (hecho habitual entre los jóvenes con elevado nivel de vida), que en este caso son costeadas mayoritariamente por la familia, y mantienen un contacto fluido con amistades y familiares de fuera del hogar. Tan solo la participación en organizaciones es, en líneas generales, menos frecuente que la media de los grupos sociales juveniles, hecho que se puede imputar a su baja edad.

\section{Grupo V: La juventud descalificada: del deterioro del estado de salud al inminente riesgo de ruptura social}

Prácticamente un millón y medio de individuos de 16 a 34 años forma un grupo juvenil descalificado. Este grupo esta caracterizado por sufrir el mayor riesgo de pobreza, una gran fragilidad de los vínculos sociales y por agrupar la práctica totalidad de individuos con problemas de salud. Nueve de cada diez jóvenes que declaran tener un estado de salud malo o muy malo, y nueve de cada diez jóvenes inactivos por incapacitación pertenecen a este grupo social. De hecho, tan solo 3 de cada 10 jóvenes declara tener un estado de salud bueno o muy bueno, cuando en los otros grupos sociales juveniles el porcentaje es siempre superior al $90 \%$.

Con una media de edad elevada (28 años), esta clase social juvenil está formada por individuos en proceso de emancipación (tiene un equilibrio casi perfecto entre jóvenes emancipados y no emancipados) y con una distribución geográfica y urbanística prácticamente idéntica a la media. La salida del hogar de origen está plenamente ligada a la pareja y a una rápida reproducción: 8 de cada 10 emancipados vive en pareja y 6 de cada 10 ya tiene hijos. La presencia de jóvenes de nacionalidad extranjera ya es importante, con un $12,5 \%$ del total.

El nivel formativo de estos jóvenes presenta claras deficiencias. Uno de cada cinco $(19,5 \%)$ tiene en la educación primaria su máximo nivel formativo, el doble que la media. Además, el 85,1\% de los jóvenes del grupo no está estudiando, y -a causa de su elevada edad y de las responsabilidades ligadas a la creación de una familia- ya difícilmente lo hará. La salida del sistema formativo se dio en paralelo a la recuperación de la economía española de mediados de los noventa, cuando estos jóvenes apenas tenían, de media, 17,6 años. A este proceso de crecimiento económico contribuyeron ellos mediante su explotación laboral, representando una mano de obra de bajo coste para los empleadores y perdiendo paulatinamente las oportunidades de acceder a ocupaciones más cualificadas.

La rápida entrada al mercado laboral y su elevada edad media hacen que estos jóvenes tengan una dilatada experiencia laboral, la cual no les supone, sin embargo, ningún rendimiento evidente. Tan solo un $56,7 \%$ de los jóvenes está ocupado (en las mujeres, un 49,6\%) y, de éstos, un 13,1\% lo hace a tiempo parcial y un $55,5 \%$ con contrato temporal. Entre los ocupados, uno de cada tres lo 
hacía como mano de obra no cualificada. Además, un 14,4\% de los jóvenes estaba desocupado y un $28,8 \%$ son inactivos, destacando la presencia de jóvenes incapacitados: representan un 6,1\% del total de jóvenes del grupo y prácticamente uno de cada cinco inactivos. En este sentido, destaca el hecho que entre los jóvenes descalificados haya 56 incapacitados por cada 100 estudiantes, cuando en el resto de grupos la relación es de 3 a 100.

Las deficiencias formativas, la precariedad laboral y el mal estado de salud tienen un reflejo en el riesgo de pobreza. Un $26 \%$ de los jóvenes descalificados sufría pobreza, un porcentaje más próximo al de los grupos poblacionales excluidos del mercado de trabajo (infancia y jubilados) que de la media de los grupos sociales juveniles. Además, este grupo disfruta de un escaso efecto de las prestaciones públicas: tan solo permiten reducir la pobreza en un $35,2 \%$, el segundo efecto más leve, aunque sí se muestran más efectivas para paliar los casos de pobreza extrema. Este factor estaría ligado a las dificultades para acceder a los sistemas de protección de tipo contributivo a causa de su débil vinculación con el mercado laboral.

La pobreza es extremadamente generalizada en los perfiles de mayor riesgo (formación básica, sin cualificación laboral, nacionalidad no europea) y es notable en los perfiles de mayor protección (hombres, mayores de 25 años, jóvenes con formación universitaria). En esta clase social aparece la mayor tasa de pobreza masculina $(24,1 \%)$, pero las mujeres siguen teniendo, también aquí, una probabilidad mayor de sufrir pobreza (27,9\%), especialmente aquellas ocupadas en tareas domésticas $(59,4 \%)$, con baja formación (49\%) y las ocupadas en empleos poco cualificados $(36,9 \%)$.

La vulnerabilidad económica se traduce en limitaciones en el ámbito de la vivienda y en el consumo. Dos de cada tres jóvenes de este grupo social se ven afectados por pobreza económica o por privaciones de carácter grave. De hecho, la pobreza es un sinónimo casi automático de privación multidimensional: tres de cada cuatro jóvenes en situación de pobreza sufren, también, privaciones graves. Así, en un 69,1\% de los casos de pobreza los gastos en el consumo representan una carga pesada para el hogar, un $66 \%$ no podría hacer frente a gastos imprevistos, un 43,5\% habita en viviendas con problemas estructurales (goteras, humidad, podredumbre), un $26,4 \%$ vive en hogares que han sufrido retrasos en el pago del alquiler o la hipoteca, y un 19,3\% no puede mantener una temperatura confortable en el hogar en invierno.

Pero, aun cuando las problemáticas económicas y materiales son dramáticas, la dimensión que refleja en mayor medida el grado de vulnerabilidad social de estos jóvenes es el riesgo de ruptura del vínculo social y relacional. Entre una tercera y una quinta parte de estos jóvenes afirma que sus encuentros y contactos con amigos y familiares de fuera del hogar no van más allá de alguna vez al año, una reclusión que en el resto de grupos sociales juveniles es prácticamente indetectable. La pobreza agravia esta ruptura social: hasta un 6,5\% de los jóvenes en situación de pobreza ha roto completamente los vínculos con familiares y amigos (no contacta ni se reúne nunca con ellos a lo largo del año), y un 25\%

EMPIRIA. Revista de Metodología de Ciencias Sociales. N. ${ }^{\circ}$ 21, enero-junio, 2011, pp. 97-120 ISSN: $1139-5737$ 
declara que no podría pedir ayuda a familiares, vecinos o amigos en caso de necesitarla. La participación en actividades públicas también cae a niveles mínimos: uno de cada cuatro jóvenes pobres $(24,8 \%)$ no había asistido a ningún tipo de actividad cultural o deportiva en el último año (11,8\% entre los no pobres), siendo la asistencia al cine la única actividad con cierta aceptación. Por último, la participación en organizaciones es prácticamente nula y tan solo las orientadas al ocio y las religiosas consiguen atraer a más de un $5 \%$ de los jóvenes, unos porcentajes muy lejanos a la media juvenil.

\section{Grupo VI: La juventud adulta precarizada: de la explotación laboral a la pobreza generacional}

Poco más de un millón de jóvenes $(9,9 \%$ del total) forman parte de una generación perdida. Esta denominación se sustenta en la constatación que estos jóvenes no se han podido beneficiar del periodo de expansión del sistema formativo característico de la juventud en los últimos años (por el contrario, en muchos casos les ha perjudicado, pues han tenido que competir en el mercado laboral con otros jóvenes mucho más formados) ni tampoco han podido transformar su experiencia laboral en una buena posición en el mercado laboral, en un contexto caracterizado por el crecimiento económico.

Esta clase social juvenil está formada por jóvenes adultos, nacidos en el último tramo del período de explosión demográfica de los años setenta. A este contingente de autóctonos ha sido agregado automáticamente un volumen importante de jóvenes de nacionalidad extranjera ( $22 \%$ del total del grupo), con una edad media bastante inferior, lo que indicaría que los ritmos de transición a la vida adulta son más prematuros entre la población de nacionalidad extranjera.

Prácticamente todos los jóvenes se han emancipado y más de la mitad están casados $(52,5 \%)$, siendo destacable la presencia de situaciones prematuras de ruptura familiar (el 3,1\% del total de jóvenes del grupo está separado, divorciado o es viudo).

Estos jóvenes no formaron parte del contingente de jóvenes universitarios de los años noventa, como sí hicieron los jóvenes adultos prósperos (con una edad similar y, posiblemente, con quienes compartieron aulas en la educación primaria). En consecuencia, este es el grupo con un menor nivel formativo, debilidad que se convierte en estructural debido a su avanzada edad. Un 16,2\% de estos jóvenes no han ido más allá de la educación primaria y cuatro de cada diez tiene en la secundaria básica su máximo nivel formativo. Además, nueve de cada diez ya no estaba cursando ningún tipo de formación. Su salida prematura salida del sistema formativo estuvo ligada a la obtención de un trabajo (el decalaje temporal entre la salida de uno y la entrada al otro es mínimo) pero, aún así, la experiencia laboral acumulada no les permite disfrutar de una posición estable. La tasa de ocupación es del 65,3\% (53,3\% en las mujeres), existe una elevada presencia de la ocupación a tiempo parcial (13,6\% del total de jóvenes, mayorita-

EMPIRIA. Revista de Metodología de Ciencias Sociales. N. ${ }^{\circ}$ 21, enero-junio, 2011, pp. 97-120. ISSN: 1139-5737 
riamente mujeres), una elevada tasa de paro (16,5\% de la población activa, aumentando hasta una de cada cuatro en el caso de las mujeres) y una generalización de las ocupaciones no cualificadas (en cuatro de cada diez jóvenes, dimensión que no mejora con la edad). Además, una de cada cinco mujeres (19,9\%) del grupo se dedicaba a las tareas del hogar, duplicando la media femenina juvenil. Esta reclusión hacia la actividad reproductiva se agudiza entre las mujeres que viven con la pareja y con hijos: el 31,7\% de ellas se dedicaba al hogar, casi tantas como las que trabajaban en una actividad productiva remunerada $(38,8 \%)$.

Por lo que se refiere a la pobreza, un $21,2 \%$ de estos jóvenes son pobres. Un índice seis puntos superior a la media juvenil pero que no refleja su baja cualificación formativa ni su precaria posición en el mercado laboral. Esto se debe especialmente a la estructura demográfica (la mayoría de estos jóvenes pertenecen a la franja adulta de edad, la más protegida ante la pobreza) y a las tipologías de hogar constituidas (uno de cada cuatro jóvenes está emancipado y vive en pareja y sin hijos). Fuera de estos perfiles protegidos y mayoritarios, el riesgo de pobreza es dramático. Estos jóvenes también sufren la forma de pobreza más intensa (aquellos que son pobres lo son con extrema gravedad), mientras que el efecto de la protección pública es escaso (tan solo reduce el riesgo de pobreza en un 31,3\%), un factor característico de los grupos de jóvenes emancipados.

La privación multidimensional también está presente y se muestra en gran medida independiente de la pobreza monetaria: el $91 \%$ de los jóvenes pobres y más del $70 \%$ de los no pobres sufrían privaciones de carácter grave en su vivienda y en el ámbito del consumo. Destaca, por ejemplo, que los gastos en vivienda y de consumo sean vistos generalizadamente como una carga pesada, que un $57,6 \%$ declaren que no podrían irse de vacaciones fuera del hogar por lo menos una semana al año o que alrededor de uno de cada cinco jóvenes haya accedido a hogares que presentan problemáticas (ruido proveniente del exterior, problemas estructurales, contaminación, delincuencia en el entorno, falta de luz en alguna habitación o dificultades para mantener una temperatura confortable en invierno.

Por lo que refiere al vínculo social y relacional constatamos la existencia de una concentración del tiempo libre hacia la familia, las amistades y la participación en organizaciones, relegando la asistencia a actividades culturales o deportivas a momentos esporádicos.

Estamos, pues, ante unos jóvenes que se insertaron en el mercado laboral justo en el fin de su contracción a mediados de los noventa y que formaron la base juvenil de la clase trabajadora que, a base de explotación y precariedad laboral, facilitó el crecimiento económico de los siguientes 15 años. La vulnerabilidad que incide sobre estos jóvenes presenta indicios de convertirse en generacional: entre los más adultos el nivel de vida no mejora y, en determinados indicadores, incluso se agravia.

La mayoría de los jóvenes de este grupo social no se contemplan en las investigaciones sobre jóvenes que conciben la juventud como una franja de edad (que habitualmente ponen el límite en los 29 años) ni en las investigaciones que

EMPIRIA. Revista de Metodología de Ciencias Sociales. N. ${ }^{\circ}$ 21, enero-junio, 2011, pp. 97-120 ISSN: $1139-5737$ 
la conciben como un periodo de transición que finaliza con la emancipación familiar del sujeto «joven». Sin embargo, la construcción de este grupo demuestra que existe en España un colectivo relativamente homogéneo de jóvenes emancipados y de edad avanzada que no se benefician de la centralidad social con la que se viene a definir la adultez.

\section{Grupo VII: La elite juvenil: acomodación social e igualdad de géneros}

A su mediana edad (nacidos a finales de los setenta y principios de los ochenta), el grupo más minoritario (poco más de un millón de jóvenes, el 9,5\% del total) integra a jóvenes que han conseguido situarse en los peldaños más elevados de la escala formativa y laboral. Jóvenes que entre finales de la década de los noventa y principios de siglo completaron su carrera formativa de larga duración y que se han insertado con éxito en el segmento primario del mercado de trabajo, sacando provecho, además, del contexto de crecimiento económico. Todos ellos viven en el hogar de origen, siendo el perfil de jóvenes no emancipado de mayor edad. El alargamiento del período formativo es la base explicativa de ello. Además, todos tienen nacionalidad española y la mayoría viven en zonas altamente urbanas (prácticamente seis de cada diez) de las CCAA con mayor desarrollo económico (Cataluña y Madrid están a la cabeza).

El 83\% de estos jóvenes ha completado estudios universitarios y el impacto de su cualificación en el mercado laboral es directo. Aún cuando su antigüedad laboral es escasa ya dominan ciertos indicadores de calidad en el trabajo: a) presentan la mayor tasa de ocupación, del 82,9\% (del 83,7\% en las mujeres, siendo el único grupo social juvenil donde la tasa de ocupación femenina es superior a la masculina), con unos máximos superiores al $90 \%$ a partir de los 25 años; b) el paro es circunstancial, y solamente afecta al 2,1\% de los jóvenes (1,9\% en las mujeres); c) el 50,3\% tenía un contrato fijo, segundo porcentaje más elevado, y d) tres de cada diez jóvenes formaba parte de las categorías profesionales dominantes: directivos, propietarios o técnicos cualificados, mientras que la vinculación con tareas no cualificadas es prácticamente inexistente (5,9\% de los jóvenes). Cabe mencionar, por el contrario, que son los jóvenes que sufren en mayor medida el fenómeno de la subocupación: su posición dominenate en el mercado laboral aún no refleja el nivel de capacitación formativa que han alcanzado.

Con estas garantías, el nivel de vida de estos jóvenes es elevado y la pobreza es casi indetectable. La tasa de pobreza se reduce al 7,6\% del total de jóvenes (7,4\% en las mujeres, la tasa de pobreza femenina más baja), y adquiere rasgos de transitoria: se limita casi exclusivamente a situaciones en las que el joven está estudiando. Además de excepcionales, las situaciones de pobreza son de muy baja intensidad.

La privación multidimensional tampoco forma parte de la cotidianeidad de estos jóvenes. Casi ocho de cada diez jóvenes no sufren privaciones graves en su hogar, que siempre están desvinculadas de situaciones de pobreza. Las priva-

EMPIRIA. Revista de Metodología de Ciencias Sociales. N. ${ }^{\circ}$ 21, enero-junio, 2011, pp. 97-120. ISSN: $1139-5737$ 
ciones más habituales en los hogares de este grupo social juvenil son aquellas más generalizadas (la carga que suponen los gastos de vivienda y de consumo) y aquellas relacionadas con el entorno de la vivienda, característica ligada al componente urbano de este grupo.

El vínculo social es, además, el más consolidado de todos los grupos sociales juveniles, pues estos jóvenes aúnan dos de las características más favorables para ello: convivencia en el hogar de origen y elevado nivel de vida, que se traducen en la acumulación de un gran volumen de capital cultural. La asistencia a actividades de tipo cultural es claramente más habitual que la media (el doble, en el caso de la asistencia a actividades como el teatro o conciertos o en las visitas a lugares de interés cultural) y la participación en organizaciones formales de todo tipo también esté generalizada, con lo que estos jóvenes consiguen ampliar su dominio en el ámbito formativo, laboral y económico también a los campos cultural, participativo y relacional.

\section{CONCLUSIÓN: LA NECESIDAD DE UN ENFOQUE METODOLÓGICO QUE ROMPA EL NOMINALISMO JUVENIL}

Mediante la clasificación de los individuos en agrupaciones sociales se ha detectado la existencia de tres grandes realidades sociales juveniles. La primera está formada por casi la mitad de los jóvenes españoles, que se encuentran en situación de protección ante la vulnerabilidad (jóvenes adultos acomodados, adolescentes urbanos acomodados y elite juvenil). Se caracterizan, con sus propias especificidades (básicamente la edad, el hecho de estudiar o trabajar, y el hecho de vivir en el hogar de origen o estar emancipados), por una ausencia de riesgo social en todas las dimensiones analizadas. En un polo opuesto, otra gran agrupación social de jóvenes, a la cual pertenece poco más de un tercio de los jóvenes (adolescentes desfavorecidos, juventud descalificada y juventud adulta precarizada) comparte una posición de debilidad en la estructura social española, que se traduce en una salida rápida del sistema educativo, una inserción precaria al mercado laboral, con una generalización de la rotación laboral, del paro, de la inactividad y de la baja cualificación, unas bajas rentas familiares, la existencia habitual de deficiencias en relación a la calidad de la vivienda así como problemas en la capacidad de consumo. También poseen, ciertamente, rasgos diferenciadores (la edad, haber finalizado o no la etapa educativa, vivir o no en el hogar de origen, el estado de salud, el acceso a la protección social y la fortaleza del vínculo social -consecuencia, en gran parte, de las anteriores-), que se deben tener presentes para evitar hablar con demasiada simplicidad de una dicotomía vulnerabilidad / no vulnerabilidad. En último lugar, una tercera gran agrupación de la juventud española está caracterizada por el contexto rural, con bajas rentas pero sin que ello se traduzca en una acumulación de limitaciones en el resto de dimensiones (aunque estas limitaciones sí aparecen en las mujeres jóvenes, especialmente en el ámbito laboral).

EMPIRIA. Revista de Metodología de Ciencias Sociales. N. ${ }^{\circ}$ 21, enero-junio, 2011, pp. 97-120 ISSN: $1139-5737$ 
El análisis que se ha presentado en este artículo resulta, pues, poco ortodoxo en el contexto de los estudios sobre la posición en la estructura social de aquello que se viene a denominar juventud. No aparecen en él datos referentes a una juventud ni comparaciones de los resultados de ésta con respecto a la infancia, adultez o vejez.

A la vista de los resultados, entendemos que hubiera supuesto someter bajo una misma categoría nominal unas realidades sociales que poco tienen en común más allá de una edad biológica similar de sus individuos, o de la realización del mismo pasaje hacía el mercado laboral y a la emancipación familiar, ignorando la estructuración de la sociedad en grupos sociales. Grupos que generan unas condiciones de existencia diferenciadas y que ofrecen un abanico heterogéneo de posibilidades y de restricciones que, en el caso que nos ocupa, generan distintas relaciones de los jóvenes con respecto a las formas vulnerabilidad social, como se ha demostrado.

Cuadro 1. Factores de vulnerabilidad social en las agrupaciones sociales juveniles, ordenados en función de la jerarquía estatus-pobreza. España, 2006 ${ }^{14}$

Factores de vulnerabilidad

\begin{tabular}{|c|c|c|c|c|c|c|}
\hline Grupo social juvenil & $\begin{array}{c}\text { Porcentaje } \\
\text { de } \\
\text { población }\end{array}$ & Formativa & Laboral & $\begin{array}{c}\text { Monetaria } \\
\text { (Pobreza) }\end{array}$ & $\begin{array}{c}\text { Material } \\
\text { (Privación) }\end{array}$ & $\begin{array}{c}\text { Vínculo } \\
\text { social }\end{array}$ \\
\hline Juventud descalificada & $11,3 \%$ & + & + & + & + & + \\
\hline Juventud adulta precarizada & $9,4 \%$ & + & + & + & + & $+/{ }_{-15}$ \\
\hline Adolescencia desfavorecida & $15,3 \%$ & + & + & + & + & - \\
\hline Adolescencia y juventud rural & $15,7 \%$ & $+/-16$ & + & + & - & - \\
\hline Adolescencia urbana acomodada & $13,7 \%$ & - & + & - & - & - \\
\hline Juventud adulta acomodada & $24,2 \%$ & - & - & - & - & - \\
\hline Élite juvenil & $9,5 \%$ & - & - & - & - & - \\
\hline
\end{tabular}

Fuente: Elaboración propia a partir de la Encuesta de Condiciones de Vida 2006

${ }^{14}$ Las casillas marcadas con un signo negativo (-) son indicativas de ausencia de vulnerabilidad. Aquellas con símbolo positivo indican presencia de vulnerabilidad en aquella dimensión. Las casillas con los dos signos (+/ -) son indicativas de ambigüedad.

${ }^{15}$ Relación ambigua con el vínculo social: existe una baja participación en actividades culturales pero no hay una ruptura del vínculo familiar o de amistades, ni de la participación en organizaciones formales.

${ }^{16}$ Se detecta un bajo nivel formativo pero es de carácter provisional: la mitad de los jóvenes de este grupo social estaban estudiando.

EMPIRIA. Revista de Metodología de Ciencias Sociales. N. ${ }^{\circ}$ 21, enero-junio, 2011, pp. 97-120. ISSN: $1139-5737$ 


\section{BIBLIOGRAFIA}

Adiego, M. y Moneo, C. (2004): Pobreza y pobreza persistente en España. 1994-2001, Madrid, Instituto Nacional de Estadística.

AlBAIGÉs, B. (2003): Crisi del treball i emergència de noves formes de subjectivitat laboral en els joves, Barcelona, Secretaria General de Joventut.

Albert, C. y Davia, M. A. (2007): Pobreza y privación juvenil en España. Propuesta de comunicación para el Encuentro de Economía Laboral y de la Educación, Gran Canaria.

AYALA, L. (coord.) (2008): «Desigualdad, pobreza y privación», en Informe FOESSA sobre la situación social en España, ed. Víctor Renes, Madrid, Fundación FOESSA / Caritas, pp. 87-171.

AyAla, L. y MARTíNEZ, R. (2005): «Las políticas contra la pobreza: factores de cambio y efectos sobre la igualdad», en Políticas públicas y distribución de la renta, ed. Jesús Ruíz-Huerta, Bilbao, Fundación BBVA.

Ayala, L. y NAVARRo, C. (2007): «The Dynamics of housing deprivation», Journal of Housing Economics, 16 , pp. 72-97.

AYllón, S. (2005): «Cohesió social: pobresa i privació», en Informe sobre la joventut al 2005, Barcelona, Secretaria de Joventut, pp. 271-293.

AYLlón, S. (2009): «Poverty and living arrangements among youth in Spain, 19802005», Demographic Research, 20 (17), pp. 403-434.

Ayllón, S.; Mercader-Prats, M. y Ramos, X. (2003): Pobresa monetària i privació a Catalunya a principis del segle XXI. Informe 2003, Barcelona, Fundació Un Sol Món, Caixa Catalunya.

BERGA, A. (2005): Adolescència femenina i risc social, Barcelona, Secretaria de Joventut.

Bourdieu, P. (2002): «La juventud no es más que una palabra», en Sociología y Cultura, México, Grijalbo.

CANTó, O. y MERCADER-Prats, M. (1999): Poverty among children and youth in Spain: The role of parents and youth employment status. Documento de trabajol 99.07, Barcelona, Universitat Autònoma de Barcelona, Departamento de Economía Aplicada.

CAnTó, O. y Mercader-Prats, M. (2001a): «Pobreza y familia: ¿son los jóvenes una ayuda o una carga?», Papeles de Economía Española, 88, pp. 151-165.

CAntó, O. y Mercader-Prats, M. (2001b): «Young people leaving home: The impact on the poverty of children and others in Spain», en The Dynamics of Child Poverty in Industrialised Countries, Cambridge, University Press, pp. 215-235.

CARdenal, M. E. (2006): El paso a la vida adulta. Dilemas y estrategias ante el empleo flexible, Madrid, Centro de Investigaciones Sociológicas.

CASAL, J. (1996): «Modos emergentes de transición a la vida adulta en el umbral del siglo XXI: aproximación sucesiva, precariedad y desestructuración», Revista Española de Investigaciones Sociológicas, 75, pp. 295-316.

Casal, J.; García, M.; Merino, R. y Quesada, M. A. (2004): Enquesta als joves de Catalunya 2002, Barcelona, Secretaria General de Joventut.

Casal, J.; García, M.; Merino, R. y Quesada, M. A. (2006): «Aportaciones teóricas y metodológicas a la sociología de la juventud desde la perspectiva de la transición», Papers, 79, pp. 21-48.

Casal, J.; MasjuAn, J. M. y Planas, J. (1988): «Elementos para un anàlisis sociológico de la transición a la vida adulta», Política y sociedad, 1, pp. 97-104. 
Comas, D. (2007): Las políticas de juventud en la España democràtica, Madrid, Instituto de la Juventud.

Edis, Ayala, L., Esteve, F., García, A., Muñóz, R., Renes, V. y Rodríguez, G. (1998): Las condiciones de vida de la población pobre en España. Informe general, Madrid, Fundación Foessa.

Elzo, J. (2006): Los jóvenes y la felicidad. ¿Dónde la buscan? ¿Dónde la encuentran?, Boadilla del Monte (Madrid), PPC.

Elzo , J. (coord.) (2009): Models educatius familiars a Catalunya, Barcelona, Fundació Jaume Bofill.

GómEz, C. (coord.) (2008): Informe de la Inclusión Social en España 2008, Barcelona, Fundació Caixa Catalunya.

IAcovou, M. y AAssve, A. (2007): Youth poverty in Europe, Colchester, University of Essex.

INE (2006): La pobreza y su medición: presentación de diversos métodos de obtención de medidas de pobreza, Madrid, Instituto Nacional de Estadística.

Maestro, I. y MARTíNeZ, J. (2003): «La pobreza humana y su feminización en España y las Comunidades Autónomas», REIS, 139, pp. 57-90.

Martín CRiado, E. (1997): «Los empleos y los paros de los jóvenes», Cuadernos de Relaciones Laborales, 11, pp. 173-201.

Martín CRiAdo, E. (1998): Producir la juventud: crítica a la sociología de la juventud, Tres Cantos, Istmo.

MARTíneZ, R. (2007): Renta y privación en España desde una perspectiva dinámica (documento de trabajo), Madrid, Fundación Alternativas.

MARTÍNEZ, R. y Ruíz-HuERTA, J. (2000): Income, multiple deprivation and poverty: an empirical analysis using Spanish data, Cracovia, 26 IARIW General Conference.

Martín-Guzmán, M. P., Bellido, N. y Jano, M. D. (2001): «La pobreza en España», Papeles de Economía Española, 88 , pp. 126-142.

MerCADER-PrATS, M. (2005): «La pauvreté menace les jeunes Espagnols au moment où ils s'emancipent», Économie et Statistique, 383-384-385, pp. 75-89.

Miret, P., Salvadó, A.; Serracant, P. y Martí, R. (2008): Enquesta a la joventut de Catalunya 2007. Una anàlisi de les transicions educatives, laborals, domiciliars i familiars, Barcelona, Secretaria de Joventut.

París, P., Serracant, P., Pascual, G., Martorell, E., Tintoré, M., Cardeña, E. y Gangolells, M. (2006): «La recerca sobre joventut a Catalunya», Papers, 79, pp. 285-217.

PAugam, S. (2007): Las formas elementales de la pobreza, Madrid, Alianza.

PÉREZ-MAYo, J. (2003): Measuring deprivation in Spain, Luxemburgo, IRISS Working Paper Series.

Pérez-MAYO, J. y FAJARdo, M. A. (2003): «Un método alternativo para identificar los hogares en situación de pobreza», Estadística española, Vol. 45, n. 152, pp. 115-134.

Ruíz-Huerta, J., LÓPEZ, J., AyAlA, L. y MARTínez, R. (1995): «Relaciones y contradicciones entre la distribución personal y la distribución espacial de la renta», $H a$ cienda Pública Española, 134, pp. 153-190.

SÁNCHEZ, C. y QuinTANA, N. (2005): «Les classes socials a Catalunya: desigualtats en les condicions de vida dels grups socials», en Estructura social i desigualtats a Catalunya, Barcelona, Mediterrània / Fundació Jaume Bofill, Vol. I, pp. 51-214.

Serracant, P.; FÁbregues, S. y Pujol, M. (2008): «Joventut i individualització. Una comparativa intergeneracional de les desigualtats per gènere $\mathrm{i}$ origen social», en

EMPIRIA. Revista de Metodología de Ciencias Sociales. N. ${ }^{\circ}$ 21, enero-junio, 2011, pp. 97-120. ISSN: $1139-5737$ 
VV.AA., Condicions de vida i desigualtats a Catalunya, 2001-2005, Barcelona, Fundació Jaume Bofill / Mediterrània, Volum II, pp. 133-180.

SubirATs, M.; SÁNCHEZ, C. y DomíngueZ, M. (1992): «Grups i classes socials a la Regió Metropolitana de Barcelona», Enquesta de la Regió Metropolitana de Barcelona 1990. Condicions de vida i hàbits de la població, Informe general, Barcelona, Institut d'Estudis Metropolitans de Barcelona, vol. 5.

SubirATs, M.; SÁNCHEZ, C. y Domínguez, M. (2002): «Classes socials i estratificació», en Enquesta de la Regió de Barcelona 2000. Condicions de Vida i hàbits de la població. Informe general, VV.AA, Barcelona, Mancomunitat de Municipis de l'Àrea Metropolitana de Barcelona y Diputació de Barcelona, pp. 219-242.

TheVenot, L, (1979): «Une jeunesse difficile. Les foctions sociales du flou et de la rigueur dans les classements», Actes de la Recherche en Sciences Sociales, 26-27, pp. 3-18.

Tully, C.J., Krug, W. y SANDer, E. (2008): «Common differences: Youth Research in Europe. An analysis based on published English and Spanish journal articles», $\mathrm{Pa}$ pers, 90, pp. 105-126.

\section{RESUMEN}

El análisis que se presenta muestra la existencia de distintas formas de vulnerabilidad social distribuidas de forma desigual entre distintas agrupaciones sociales de jóvenes. Esta conclusión se sustenta en una metodología de construcción de grupos sociales juveniles que ha tenido como objetivo romper la homogeneización artificial mediante la cual se constituye habitualmente el concepto juventud. Una juventud que no se entiende aquí simplemente como un conjunto de individuos de una misma cohorte de edad sino como un agregado heterogéneo de agrupaciones de individuos que, en su doble pasaje de inserción al mercado laboral y de emancipación familiar, comparten unas similares condiciones sociales de existencia. Se han detectado siete tipos ideales de agrupaciones sociales juveniles en España: juventud adulta próspera, adolescencia rural, adolescencia desfavorecida, adolescencia urbana acomodada, juventud descalificada, juventud adulta precarizada, y élite juvenil. Finalmente, hemos analizado cómo cada una de estas agrupaciones sociales juveniles presenta una morfología específica de vulnerabilidad.

\section{PALABRAS CLAVE}

Pobreza, juventud, exclusión social, desigualdad. 


\begin{abstract}
The analysis presented shows the existence of different forms of social vulnerability distributed heterogeneously among different young social groups. This assertion is sustained in a methodology of construction of social juvenile groups whose aim was to break the artificial homogenization usually used for the concept of youth. Youth is not considered here simply as a set of individuals who share the same cohort of age, but as a heterogeneous group of individuals who share similar social conditions of existence in their insertion to the labour market and familiar emancipation. The result has been the detection of seven ideal types of juvenile social groups in Spain: young prosperous adults, rural adolescents, disadvantaged adolescents, wealthy urban teens, disqualified youth, young precarious adults, and juvenile elite. Finally, we have analyzed how each one of these juvenile groups presents a specific morphology of vulnerability.
\end{abstract}

\title{
KEYBOARDS
}

Poverty, youth, social exclusion, inequality. 